\title{
Biodiversidad de gasterópodos terrestres (Mollusca) en el Parque Biológico Sierra de San Javier, Tucumán, Argentina
}

\author{
María José Miranda \& María Gabriela Cuezzo \\ CONICET-Facultad de Ciencias Naturales, Universidad Nacional de Tucumán, Miguel Lillo 251, 4000 Tucumán, \\ Argentina; mirandamjo@hotmail.com, mcuezzo@unt.edu.ar
}

Recibido 08-III-2009. Corregido 12-III-2010. Aceptado 08-IV-2010.

\begin{abstract}
Biodiversity of land gastropods (Mollusca) in Sierra de San Javier Park, Tucumán, Argentina. Studies related to land mollusk diversity in tropical and subtropical forests are scarce. To assess this, a study on land snail diversity of subtropical cloudforest (Yungas) and dry forest (Chaco) areas of Sierra de San Javier Park, Tucumán, Argentina, was carried out. Taxonomic identifications were performed to species level and built a species per stations data matrix to analyze diversity patterns on qualitative and quantitative samples processed from 10x10m quadrates in altitudinal transects. Non parametric analysis (ICE, ACE, Chao 1 and Chao 2) were used to estimate the true diversity of the area, as well as the degree of undersampling and spatial aggregation of the data. Diversity was also calculated using Shannon, Simpson, Whittaker and Jaccard indices. The richness of the San Javier Park was estimated to be 32 species distributed into 13 families and 21 genera. From the total number of species collected, a single one belongs to Caenogastropoda, while the rest of the species are classified into Pulmonata Stylommatophora and Systellommatophora. The most representative family was the micromollusc Charopidae, while the most relatively abundant species was another micromollusc snail, Adelopoma tucma. Richness and diversity were slightly more elevated in dry forest areas of the Chacoan Ecoregion than in cloud forest areas of Yungas. Non parametric estimators showed that the inventory was complete. Diversity values obtained were high in comparison to previously studied areas of Northwestern Argentina. The total number of specimen collected (22 169 specimens), was higher than other published studies. Rev. Biol. Trop. 58 (3): 10091029. Epub 2010 September 01.
\end{abstract}

Key words: Stylommatophora, Systellomatophora, Yungas, Chacoan region, micromolluscs, macromolluscs, abundance.

La Provincia de Tucumán posee formaciones boscosas correspondientes a las regiones Chaqueña, Selva nublada (Yungas) y Monte (Cabrera 1976, Vides Almonacid et al. 1998, Morrone 2004). Las Yungas en Argentina se extienden desde la frontera con Bolivia $\left(23^{\circ} \mathrm{S}\right)$ hasta el Norte de Catamarca $\left(29^{\circ} \mathrm{S}\right)$ por el oeste de las provincias de Salta, Jujuy y Tucumán. La otra formación de selva subtropical que existe en Argentina es la selva Paranaense (provincia de Misiones, noreste de Argentina), que junto a las Yungas constituyen menos del 2\% de la superficie de Argentina y contienen más del $50 \%$ de la biodiversidad del país (Salas Oroño et al. 2007). Estos ecosistemas húmedos sufren un proceso acelerado de transformación debido a la deforestación de las áreas pedemontanas para el desarrollo de asentamientos humanos y la conversión en áreas de agricultura y ganadería. Otra de las causas de dicha modificación es la explotación forestal e introducción de especies exóticas, y que en general constituyen una amenaza para la biodiversidad. La Provincia biogeográfica Chaqueña se extiende desde el Norte de Argentina, hasta las provincias de Córdoba, San Luis y Santa Fé (Cabrera \& Willink 1973). El Chaco Serrano es un ambiente fragmentado que se distribuye en forma 
de islas desde Salta hasta Córdoba (Vides Almonacid et al. 1998). El bosque Chaqueño se encuentra muy alterado por la explotación forestal y la ganadería intensiva.

El filo Mollusca representa el segundo grupo animal más diverso en cuanto a número de especies descritas, siendo superado solo por Arthropoda. Se estima que probablemente existen entre 11000 a 40000 especies de moluscos aún no descritas, lo cual puede deberse en parte a que hay regiones del mundo que permanecen inexploradas (Lydeard et al. 2004). Los gasterópodos se hallan representados por entre 40000 a 150000 especies, mientras que la riqueza global de grupos terrestres se estima entre 30000 y 35000 especies de ese total (Aktipis et al. 2008).

Los estudios referidos a la diversidad de moluscos terrestres en selvas tropicales y subtropicales son escasos. Solem (1984) consideró que los gasterópodos representaban un grupo poco diverso y abundante en áreas tropicales. Sin embargo, estudios previos realizados en distintas áreas, tales como Camerún (de Winter \& Gittenberger 1998), Madagascar (Emberton et al. 1999), Kenia (Tattersfield et al. 2001), Malasia (Schilthuizen \& Rotjes 2001), Creta (Cameron et al. 2003), Gabon (Fontaine et al. 2007) y Azores (Cameron et al. 2007), demostraron que la malacofauna terrestre de selvas tropicales puede ser tanto rica, como diversa. Sin embargo, a pesar de la importancia en términos de riqueza y diversidad que tienen los gasterópodos terrestres, estudios de este tipo en Sudamérica son escasos, abundando mayoritariamente listados de especies en áreas o por país. Entre los estudios previos realizados en Sudamérica podemos citar a Gargominy \& Ripken (1998) quienes analizaron la diversidad de moluscos en Guyana Francesa, Salas Oroño et al. (2007) en Argentina y Miquel et al. (2007) en Brasil. Salas Oroño et al. (2007), realizaron un estudio sobre diversidad de micromoluscos terrestres en selvas de montaña de la provincia de Tucumán, noroeste de Argentina. En este estudio previo se detectó una alta diversidad, particularmente en el Parque Biológico Sierra de San Javier, motivo por el cual el objetivo del presente trabajo fue realizar un estudio más detallado sobre el mismo, con mayor esfuerzo de muestreo e incluyendo los grupos de macromoluscos que no fueron considerados anteriormente en Salas Oroño et al. (2007).

La importancia del presente trabajo radica en que la obtención de datos taxonómicos y ecológicos de moluscos gasterópodos en distintos ecosistemas, importante como información base para futuros estudios de evaluación y monitoreo para contribuir con su conservación.

Los objetivos planteados fueron realizar un inventario taxonómico de la malacofauna terrestre, a fin de definir la posible existencia de una malacofauna única y característica de cada ecosistema. Estimar la diversidad por medio del cálculo de la diversidad alfa $(\alpha)$ y beta $(\beta)$ y determinar las variaciones de riqueza entre las áreas muestreadas. También se calcula la variación de distintos parámetros de diversidad entre la Selva de Yungas y el Bosque Chaqueño Serrano.

\section{MATERIALES Y MÉTODOS}

Área de estudio: El Parque Biológico Sierra de San Javier es un área natural protegida dependiente de la Universidad Nacional de Tucumán, Tucumán, Argentina. Dicha área de reserva tiene una extensión de 14 100ha, ocupando el $74 \%$ de la sierra que le da su nombre, ubicada al Oeste de San Miguel de Tucumán. El parque se extiende entre los $26^{\circ} 38^{\prime}-26^{\circ} 57^{\prime} \mathrm{S}$ y los $65^{\circ} 6^{\prime}-65^{\circ} 20^{\prime} \mathrm{W}$. Incluye las laderas norte, este y sur de la sierra, desde los 600 hasta los 1 200msnm, y un sector de bosque de pino y pastizal que forman el área cumbral desde 1 200 hasta $1850 \mathrm{~m}$. El clima de la Sierra es templado húmedo con veranos lluviosos e inviernos secos. El Parque protege las ecorregiones de Yungas (laderas más húmedas) y Bosque Chaqueño Serrano (sector NO y ladera Oeste de la sierra).

En la selva de Yungas del noroeste argentino pueden reconocerse estratos altitudinales de vegetación, la Selva Pedemontana (400$700 \mathrm{~m}$ ) con precipitación de $1000 \mathrm{~mm}$ anuales, 
con especies de árboles dominantes tales como Callycophyllum multiflorum, Griseb. (palo blanco) y Phyllostylon rhamnoides (Poisson) Taubert (palo amarillo). En el noroeste argentino dicho piso de vegetación se halla altamente modificado por la actividad humana y en la zona del Parque solo el área correspondiente a Horco Molle, situado entre 700 y $900 \mathrm{~m}$, resguarda vegetación que podría ser considerada como de selva pedemontana. El segundo nivel corresponde a la Selva Montana (700-1 $500 \mathrm{~m}$ ) de máxima precipitación entre 1500 y 3 000 $\mathrm{mm}$ anuales en la cual predominan Tipuana tipu Benth (tipa) y Cynamomun porphyrium (Griseb.) Kosterm (laurel). El tercer estrato de vegetación corresponde a los Bosques Montanos (1 500-1 700m) pudiendo llegar hasta los 3 $000 \mathrm{~m}$ con zonas de pastizales, precipitaciones de 900 a $1300 \mathrm{~mm}$ anuales. En este estrato se distingue el bosque de pino, dominado por Podocarpus parlatorei Pilger (pino del cerro) y el bosque de Alnus acuminata (Kunth) (aliso) (1 400-2 100m) (Valdora \& Soria 1999, Brown et al. 2002). En el Bosque Chaqueño Serrano la vegetación predominante es xerófila caducifolia, con un estrato herbáceo de gramíneas, cactáceas y bromeliáceas. Este ecosistema no se divide en estratos altitudinales como ocurre con las Yungas, ya que su distribución está restringida a altitudes no mayores de $1200 \mathrm{~m}$ dentro del área del parque. Abundan árboles como Schinopsis hankeana Engler (horco quebracho), Schinopsis balansae Engl. (quebracho colorado), Prosopis nigra (Grisebach) (algarrobo negro), Prosopis alba Griseb. (algarrobo blanco) y cactáceas de los géneros Opuntia, Cereus, Trichocereus, entre otros (Cabrera 1976).

Muestreo: Los muestreos fueron llevados a cabo en períodos posteriores a precipitaciones, ya que la alta humedad del ambiente permite que los gasterópodos estén más activos (Barrientos 2003). Se incluyó meses coincidentes con la época reproductiva de mayor actividad en gasterópodos (Noviembre-Abril). Se muestrearon cuatro sendas altitudinales, dos en zonas de Yungas (Y1: 26 36'24" S-65'20'03"
W y Y2: $26^{\circ} 36^{\prime} 28^{\prime \prime}$ S-65 $65^{\circ} 13^{\prime} 44^{\prime \prime}$ W) y dos en

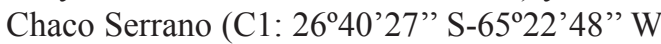
y C2: $26^{\circ} 40^{\prime} 47^{\prime \prime}$ S-65 $\left.65^{\circ} 23^{\prime} 13^{\prime \prime} \mathrm{W}\right)$. Las coordenadas geográficas (GPS Garmin), fueron registradas en el punto inicial de cada senda. A lo largo de las mismas, fueron evaluadas un total de 25 parcelas de $10 \times 10 \mathrm{~m}$ distribuidas así: $\mathrm{Y} 1=7, \mathrm{Y} 2=7, \mathrm{C} 1=7$ y $\mathrm{C} 2=4$ (debido a poca accesibilidad). Las parcelas de las sendas Yungas fueron comparadas con la Senda $\mathrm{La}$ Nina, que fuera utilizada en un estudio previo (Salas Oroño et al. 2007), y que corresponde a un sector más al Norte del Parque Sierra de San Javier. En cada una de ellas se registraron: altitud (altímetro Bushnell), número de árboles con perímetro de tronco mayor a $1 \mathrm{~m}$, porcentaje de cobertura vegetal (estimado visualmente), características del suelo (presencia o ausencia de rocas, hojarasca, palos, entre otros), grado de humedad del suelo (estimada con base en una escala cualitativa: Máxima $=$ los troncos caídos se desarman; Alta=troncos y suelo se hallan humedecidos en profundidad; Media=hojarasca húmeda, no así los troncos y tierra debajo de hojarasca, Baja=hojarasca y tierra seca) y pendiente (Cuadro 1). Las parcelas muestreadas corresponden al estrato vegetal de Selva Montana de Yungas y de Bosque Chaqueño Serrano. Debido a que el bosque chaqueño serrano en el Parque no tiene una gran variación actitudinal, las parcelas no se agruparon por altitudes similares.

En cada senda se efectuó un total de siete muestreos cualitativos (una hora) y cuantitativos, respectivamente, y para $\mathrm{C} 2$, cuatro. En cada muestreo cualitativo, se recolectaron especímenes de micromoluscos (con concha de dimensión inferior o igual a los $5 \mathrm{~mm}$ ) y macromoluscos (concha mayor a $5 \mathrm{~mm}$, etapa adulta). Para la recolección de especímenes se siguió la metodología propuesta por de Winter \& Gittenberger (1998) y adaptada por Salas Oroño et al. (2007). En el muestreo cualitativo, realizado por dos personas durante $1 \mathrm{hr}$ en cada parcela, se revisaron los microhabitats que podrían ser más adecuados para albergar gasterópodos: debajo de troncos caídos, huecos y zona entre raíces de árboles, entre otros. El muestreo 


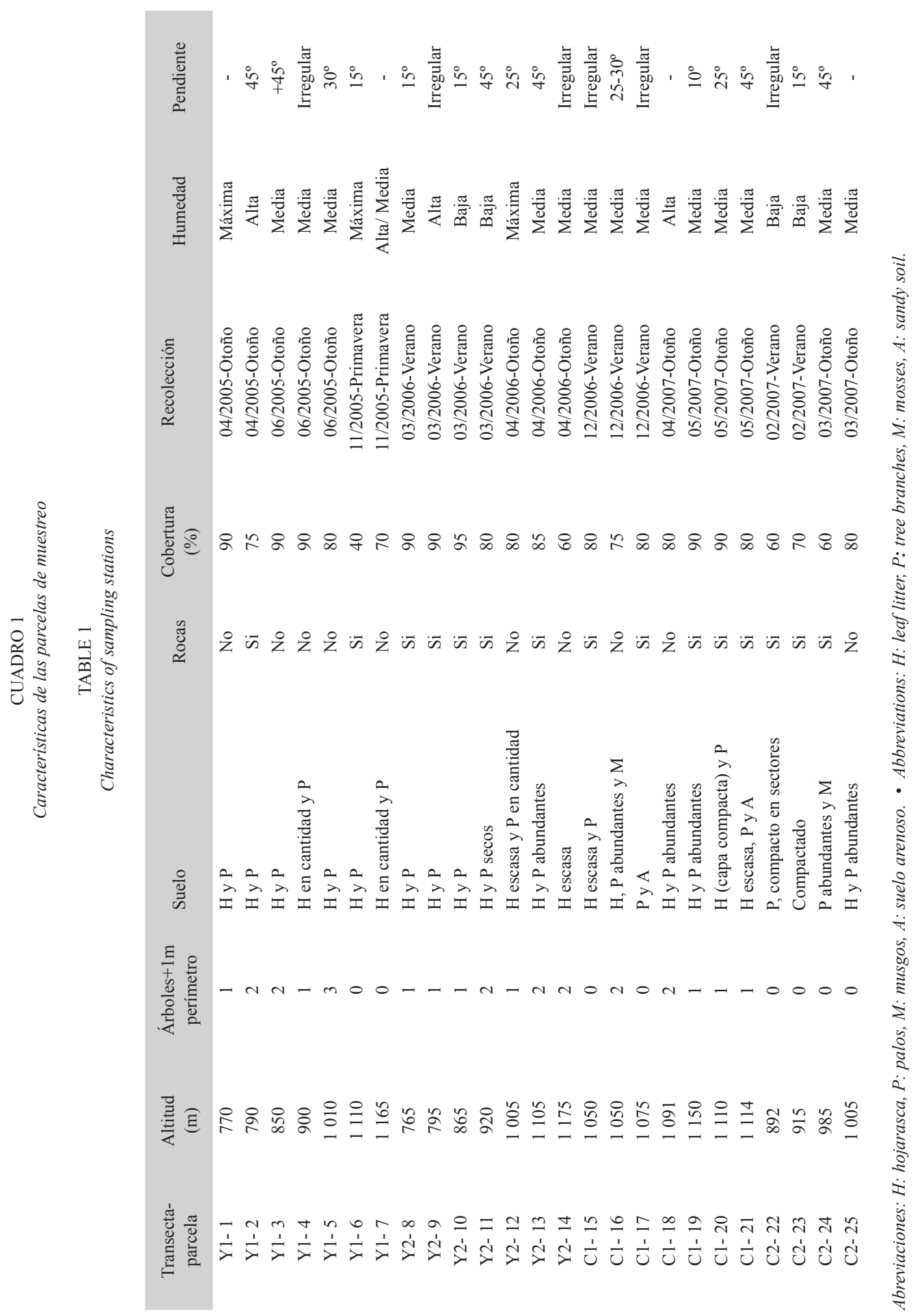


cuantitativo consistió en la toma de tres muestras (hojarasca+suelo) al azar dentro de cada parcela. Cada muestra de suelo y hojarasca se tomó de un cuadrante de $50 \times 50 \mathrm{~cm}$ y $3 \mathrm{~cm}$ de profundidad. Se recolectaron especímenes vivos y conchas vacías. El material obtenido en el muestreo cualitativo fue colocado en frascos y tubos plásticos con tapas, los que fueron etiquetados, separando previamente las conchas vacías de los ejemplares vivos, para el posterior tratamiento de éstos. En el laboratorio, el material vivo, fue fotografiado con la finalidad de conservar registros del aspecto y coloración del animal. Previo a la fijación de los especímenes vivos recolectados (micromoluscos y macromoluscos), se procedió a relajarlos en agua durante $24 \mathrm{~h}$. La fijación de los tejidos blandos se realizó en alcohol $96 \%$ durante $24 \mathrm{~h}$ y posteriormente se preservó en alcohol $70 \%$. El procedimiento de relajación permitió la realización de estudios anatómicos necesarios para la determinación taxonómica de algunas de las especies. Las muestras cuantitativas de hojarasca y suelo fueron mantenidas en las bolsas plásticas usadas para su traslado y fueron aireadas periódicamente, con el fin de evitar el exceso de humedad y la consiguiente descomposición de la materia orgánica. Las muestras fueron separadas a través de tres tamices de tamaños de malla decrecientes (4, 2 y $1 \mathrm{~mm})$, para lograr la separación en estratos de especímenes y tierra u hojarasca. Cada tamizado fue examinado bajo microscopio estereoscópico Leica MZ6, y se separaron las conchas y los organismos vivos presentes, evitando así la pérdida de especímenes que pasarían desapercibidos por su escaso tamaño

Identificación taxonómica: Se realizó con base en caracteres morfológicos de la concha, utilizando la clave de Fernández \& Castellanos (1973), comparaciones con material identificado depositado en la Colección Malacológica del Instituto Miguel Lillo (IML) y descripciones originales de las especies (Hylton Scott 1948, 1950, 1957, 1984, Weyrauch 1965). Cuando las conchas vacías recolectadas estaban rotas o muy desgastadas, fueron descartadas por no poder ser identificadas con seguridad. En todos aquellos casos en que fue posible, la identificación se hizo hasta el nivel de especie, en otros casos, se determinaron morfoespecies. En el caso de Bulimulus apodemetes (d' Orbigny 1835), Drepanostomella tucma (d' Orbigny 1835) y Phyllocaulis soleiformis (d' Orbigny 1835) se realizaron además estudios anatómicos con el fin de asegurar la correcta identificación taxonómica. El material recolectado fue depositado en la Colección Malacológica del IML. El inventario taxonómico se realizó siguiendo la clasificación suprafamiliar de Bouchet \& Rocroi (2005), y para grupos particulares, Miquel et al. (2004), Miquel \& Bellosi (2006), Miquel et al. (2007) y Hausdorf (2006, 2007) (Cuadro 2).

Matrices de datos: La matriz de incidencia (Cuadro 3) se elaboró con los datos de presencia-ausencia de las especies por parcela, sumando los muestreos cualitativos y cuantitativos. La finalidad de unificar la información reunida a través de ambos muestreos, fue la de reflejar la composición de especies presentes en cada parcela. Esto se debió a que ciertas especies fueron recolectadas exclusivamente a partir del muestreo cualitativo, mientras que otras solo se obtuvieron a través del cuantitativo. Además se incluyeron los registros de presencia-ausencia de una transecta adicional, "La Nina" (Salas Oroño et al. 2007), con fines comparativos. Se codificó así: uno para la presencia de especies y cero para la ausencia. La matriz de abundancia (Cuadro 4) incluye el número de especímenes registrados de las especies por parcela.

Análisis de diversidad: Para evaluar si el muestreo usado en este estudio es representativo de la comunidad y estimar la riqueza de gasterópodos, se empleó el programa EstimateS versión 5.0.1 (Colwell 1997). Se utilizó más de un estimador, con el propósito de comparar los valores generados a partir del análisis de los observados y su tendencia. El uso de distintos estimadores no-paramétricos permite determinar la calidad del muestreo realizado y 
CUADRO 2

Especies registradas en el Parque Biológico Sierra de San Javier, Tucumán, Argentina

TABLE 2

Species collected in the Sierra de San Javier Park, Tucumán, Argentina

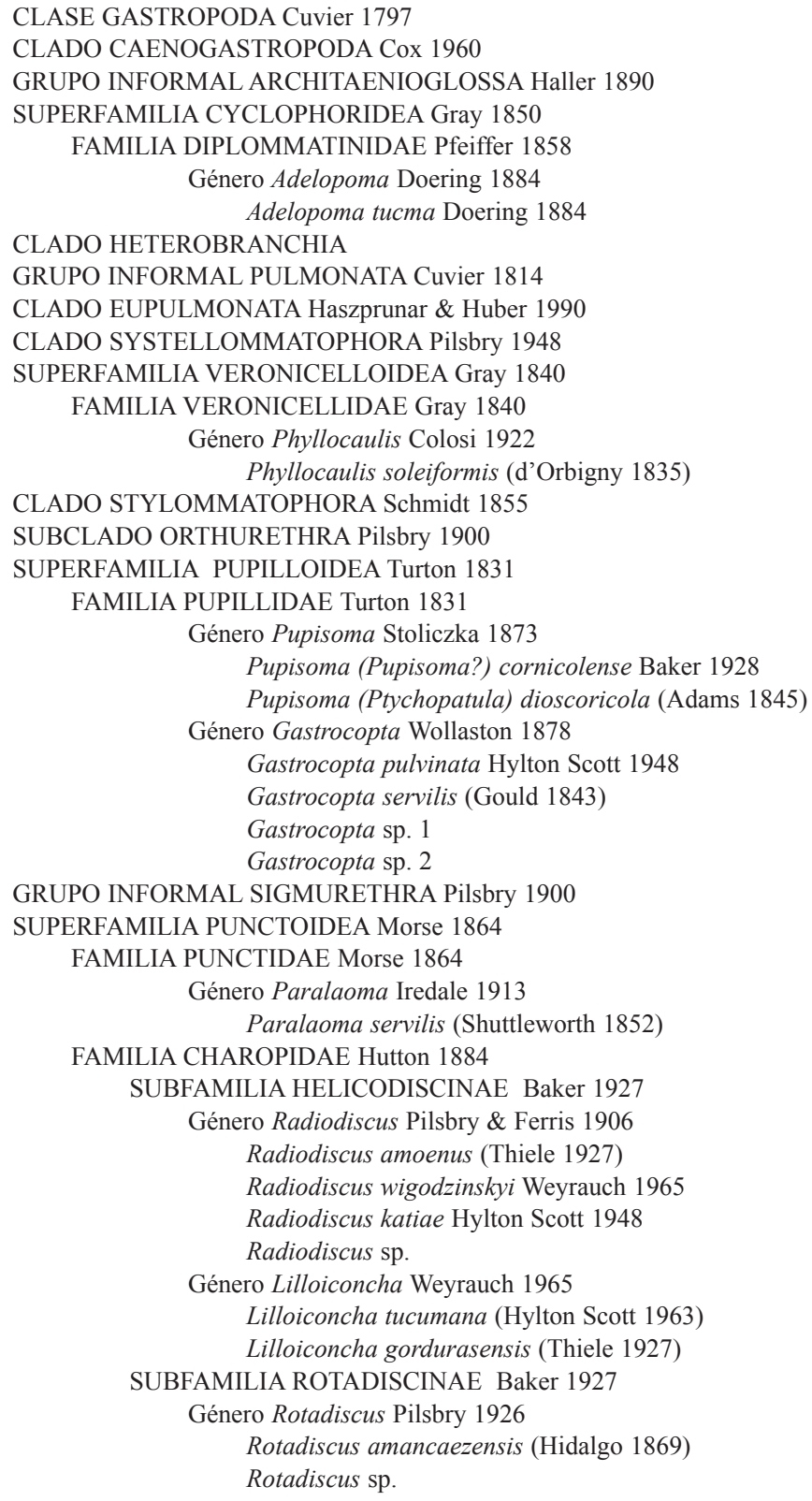


CUADRO 2 (Continuación)

Especies registradas en el Parque Biológico Sierra de San Javier, Tucumán, Argentina

TABLE 2 (Continued)

Species collected in the Sierra de San Javier Park, Tucumán, Argentina

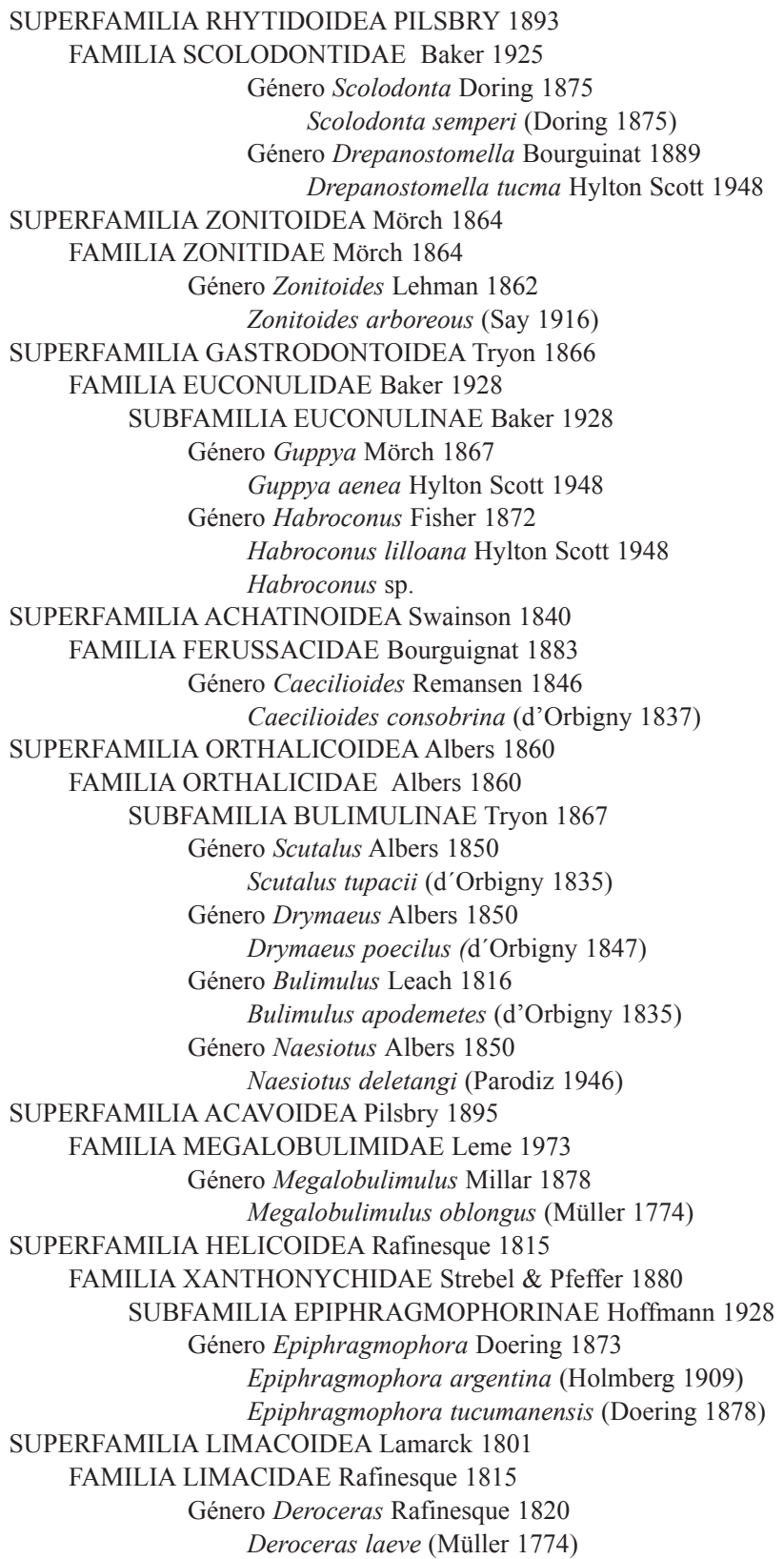




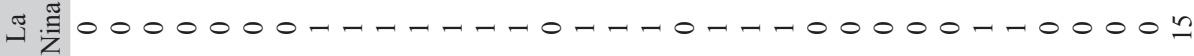
$\approx 0-0-7-00000000000000000-000-000000$ บ A $-10-7-100000000000000000-07-1000000$

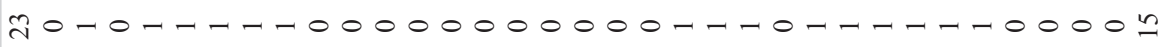
40-0-7-0-000000000000-10-0-7-70000

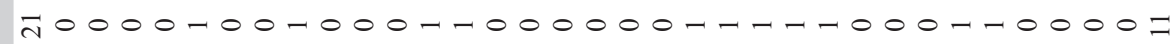
ㄱ- $-000000-0-0-1-100-1-10-0000-00000 m$ I-000000-000-10000-1-1-1000000-00000

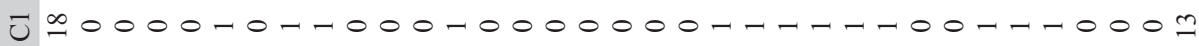
=0000-10-000-1000000-1-10-1-00-1-1000月 $10-00-00-000000000000-1-1-000-100000$ n0-00-10-000000000000-1-1-000-100000 I-000000-000-0000-1-0-0-000000-00000 mo-10000-100-0-000-1-1-100000-10-10n I-0-0000-1-0000-000-0-1-1-00000-00-0I N $=-0-0000-1-0-1-000-1-1-00000-1-0000$ $00000000-1-0-1-000-0-1-10000-1-100$ n

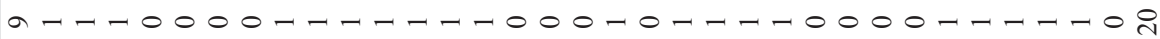

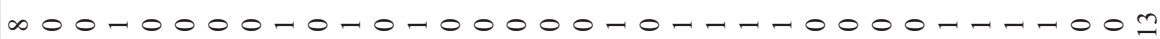
n-0-0000-1-0-1--1- - - - - - - - $00000-1000$ n $0-0-0000-1-0-1-00-\cdots-1-1000000-10000$ nn-1-10000-1-10-1-100-0-1-10000-1-1000

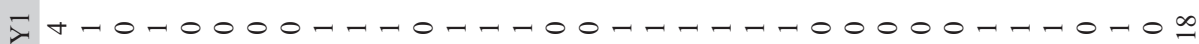

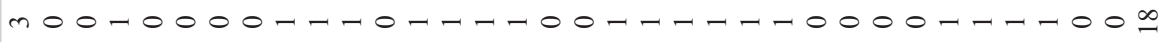
$4-7-0000-7-0-0-000-7-7-70000-7-7-109$ $---70000-7-0-7-000-7-7-00000-7-7-4$

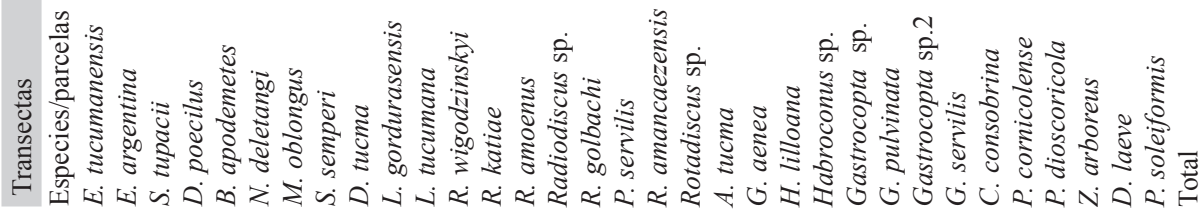




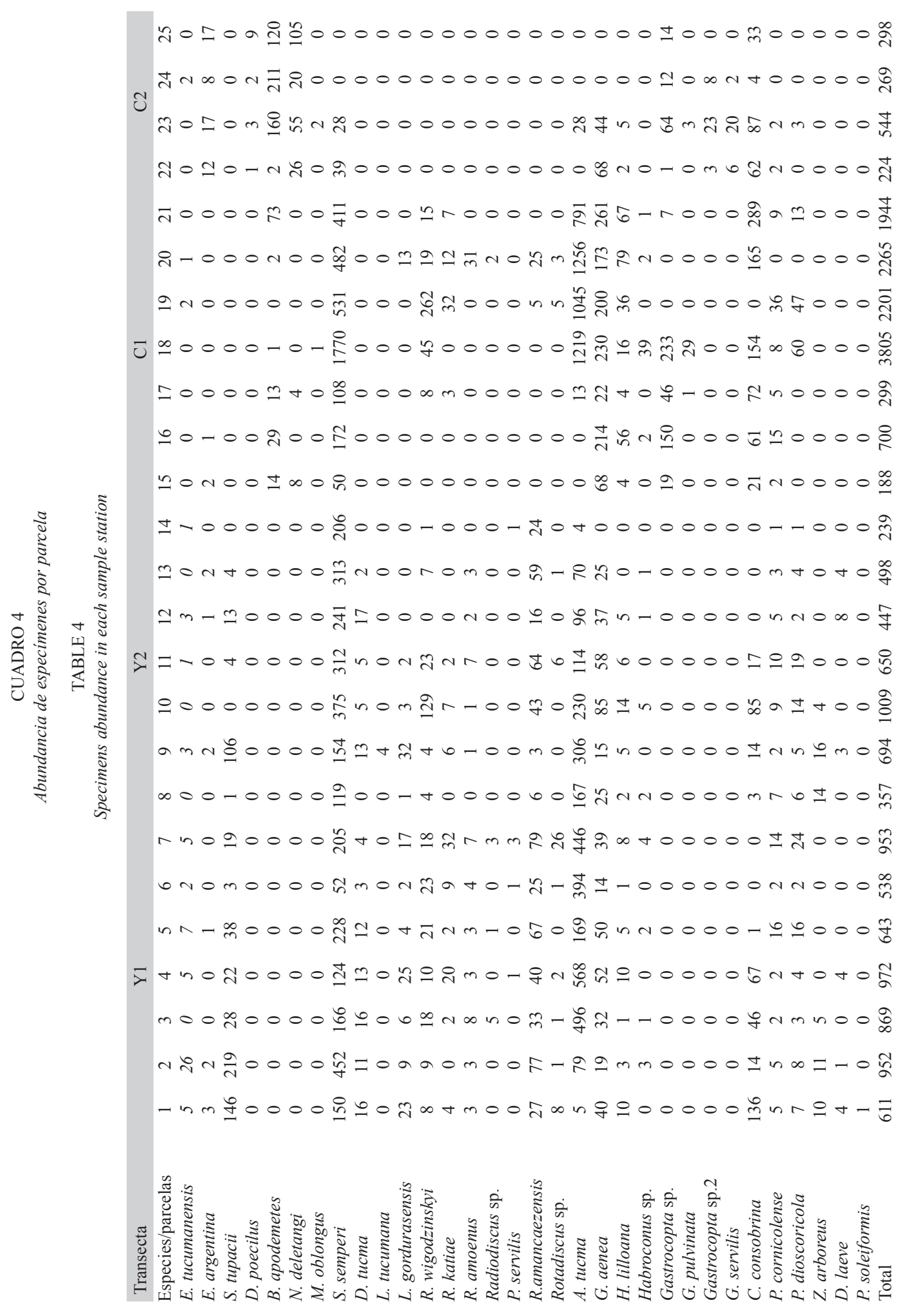


el número de especies que falta por recolectar, basándose en la cuantificación de las especies raras encontradas (Toti et al. 2000). Una especie rara es aquella que está presente solo en una o dos muestras o que está representada por uno o dos individuos en el conjunto de muestras. Para analizar la matriz de incidencia los estimadores no paramétricos seleccionados fueron Incidence-based Coverage Estimator (ICE) (Lee \& Chao 1994), para conocer el número de especies esperadas con base en las especies encontradas en diez o menos unidades de muestreo (Lee \& Chao 1994). Chao2 (Chao 1987) estima el número de especies esperadas considerando la relación entre la cantidad de especies únicas (que aparecen en una muestra) y las duplicadas (que aparecen en dos muestras). Para analizar la matriz de abundancia, se usaron los estimadores Abundance-base Coverage Estimator (ACE) (Chao et al. 1993) que calculan las especies esperadas, con base en especies con diez o menos individuos en la muestra y Chaol (Chao 1984), para saber las especies esperadas considerando la relación entre las especies representadas por un individuo (singletons) y las representadas por dos individuos en las muestras (doubletons). Además para ambas matrices se obtuvieron las curvas de Coleman (Cole) (Coleman 1981, Coleman et al. 1982) o de rarefacción para estimar la riqueza específica con base en la riqueza total de especies. Dicha curva es una herramienta importante para evaluar gráficamente el grado de agregación espacial de los datos (Heyer et al. 1999). La comparación de las curvas de acumulación de especies con la curva de Coleman, permite observar la heterogeneidad del muestreo (Colwell 1997). Se graficaron las curvas de acumulación para evaluar la calidad del muestreo así como el número probable de especies presentes en el área de estudio.

Se calcularon los índices de Shannon, Simpson, Equitatividad, Whittaker y similitud de Jaccard (de Winter \& Gittenberger 1998, Krebs 1989, 1995, Magurran 1989, Margalef 1972, Whittaker 1975).

\section{RESULTADOS}

En el área de estudio se registraron 32 especies (22 169 especímenes), 23 micromoluscos y nueve macromoluscos; clasificadas en 13 familias y 21 géneros (Cuadro 2). Del total registrado sólo una especie corresponde a Caenogastropoda, Adelopoma tucma Doering 1884, mientras que las restantes pertenecen a los pulmonados (Stylommatophora y Systellommatophora).

La riqueza obtenida con el muestreo cualitativo fue de 24 especies (nueve macromoluscos y 15 micromoluscos) mientras que con el muestreo cuantitativo se recolectaron 29 especies (cinco macro y 24 de micro). La riqueza por parcela varió entre seis y 20 especies (Cuadro 3). Las parcelas con mayor riqueza fueron la uno y la nueve, ambas con 20 especies. A su vez, la parcela con menor riqueza específica (seis especies) fue la 25. La riqueza total de la Ecorregión chaqueña asciende a 25 especies y en Yungas se registraron 24. Ambas ecorregiones comparten 17 especies. A su vez, siete especies fueron recolectadas exclusivamente en Yungas, mientras que ocho únicamente en Chaco Serrano. En Yungas se detectó una disminución de la riqueza a medida que el gradiente altitudinal aumenta. Dicha disminución fue de 20 a 15 especies en Y1 y de 13 a ocho especies en Y2. Sin embargo, en la Ecorregión chaqueña, este comportamiento se observa solo en C2 (de 12 a seis especies) (Fig. 1A). Las familias de micromoluscos con mayor riqueza en el área de estudio fueron Charopidae (siete especies) y Pupillidae (seis), mientras que la de macromoluscos es Orthalicidae (=Bulimulidae) (cuatro). Las tres familias juntas representan el $59 \%$ del total de especies. La familia Charopidae, además resultó ser la más representada en Yungas mientras que en Chaco Serrano, lo fue Pupillidae. A pesar de esta diferencia, ambas familias fueron recolectadas en las dos ecorregiones.

El número total de especímenes recolectados fue de 18567 en el muestreo cuantitativo 


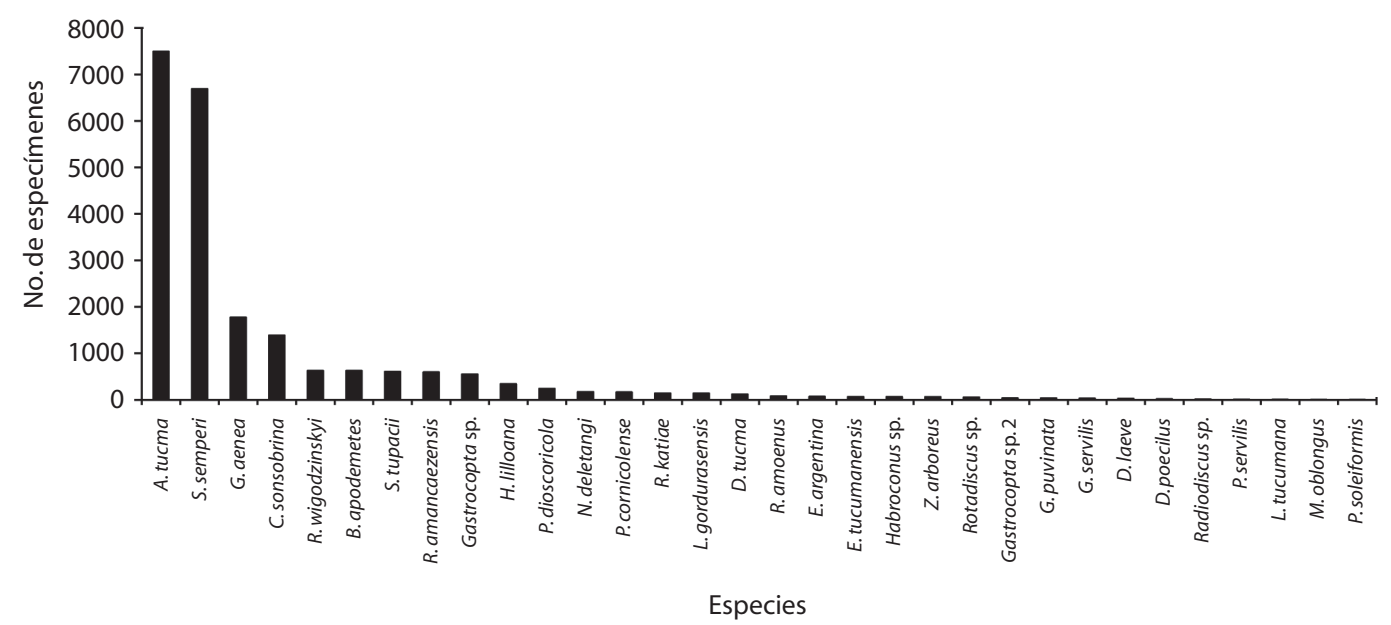

Fig. 1. (A) Variación altitudinal de la riqueza de especies en las transectas de muestreo. (B) Variaciónn altitudinal de la abundancia de especímenes en las transectas de muestreo.

Fig. 1. (A) Altitudinal variation of richness of species in the sample tracks. (B) Altitudinal variation of abundance of specimens in the sample tracks.

( $84 \%$ del total) y 3630 en el cualitativo $(16 \%)$. Con respecto al número total de especímenes por transecta recorrida, en Y1 se registraron 5538 especímenes y en Y2, 3 894. A su vez en Chaco (C1) se registraron 11402 y en $\mathrm{C} 2$, 1335 especímenes. Estos valores totalizan 12 737 especímenes para la Ecorregión Chaqueña (57\% del total) y 9432 para Yungas (43\%). La densidad varió entre 2 y 38 especímenes $/ \mathrm{m}^{2}$ en la Ecorregión Chaqueña, mientras que en Yungas osciló entre 4 y 10 especímenes $/ \mathrm{m}^{2}$.

La especie de micromoluscos con mayor abundancia relativa en el área de estudio fue A. tucma, seguida por Scolodonta semperi (Doering 1875), Guppya aenea Hylton Scott 1948 y Caecilioides consobrina, (d' Orbigny 1837) (Fig. 2). Estas especies representan el $78 \%$ del total de especímenes recolectados. Las especies de macromoluscos con mayor abundancia relativa (Fig. 2) fueron B. apodemetes y Scutalus tupacii (d' Orbigny 1835), representando el 5\% del total de los especímenes. La contribución de ambas especies de babosas, Deroceras laeve (Müller 1774) у $P$. soleiformis, fue muy baja (0.11\%). Los valores de abundancia fluctuaron a través del gradiente altitudinal, sin mostrar una tendencia definida. En la Ecorregión de Yungas, tanto en Y1 como en Y2, las parcelas ubicadas por debajo de los 900m exhibieron mayor abundancia en relación con las de mayor altitud. En cambio en C1, en las parcelas de mayor altitud se dio una mayor abundancia (Fig. 1B).

S. semperi fue la especie más frecuente en las parcelas muestreadas ( $92 \%$ de ocurrencia). A su vez, G. aenea y Habroconus lilloana (Hylton Scott 1948), también tuvieron una alta frecuencia de ocurrencia (88\%). Por otra parte, las especies menos frecuentes fueron Lilloiconcha tucumana (Hylton Scott 1963) y P. soleiformis (4\% para ambas especies) (Fig. 3).

La riqueza específica con los estimadores ACE y Chaol fue de 32 especies, de 31 para Chao2 y 34 para ICE (Figs. 4, 5). El comportamiento de los estimadores basados en datos de incidencia, con respecto a la curva de acumulación de especies se muestra en la Fig. 4. La curva de acumulación de especies observadas (Sobs) presentó un crecimiento continuo hasta estabilizarse finalmente en una asíntota. La curva del estimador ICE se aproxima a la curva de Sobs, sin llegar a unirse a ella. La trayectoria 

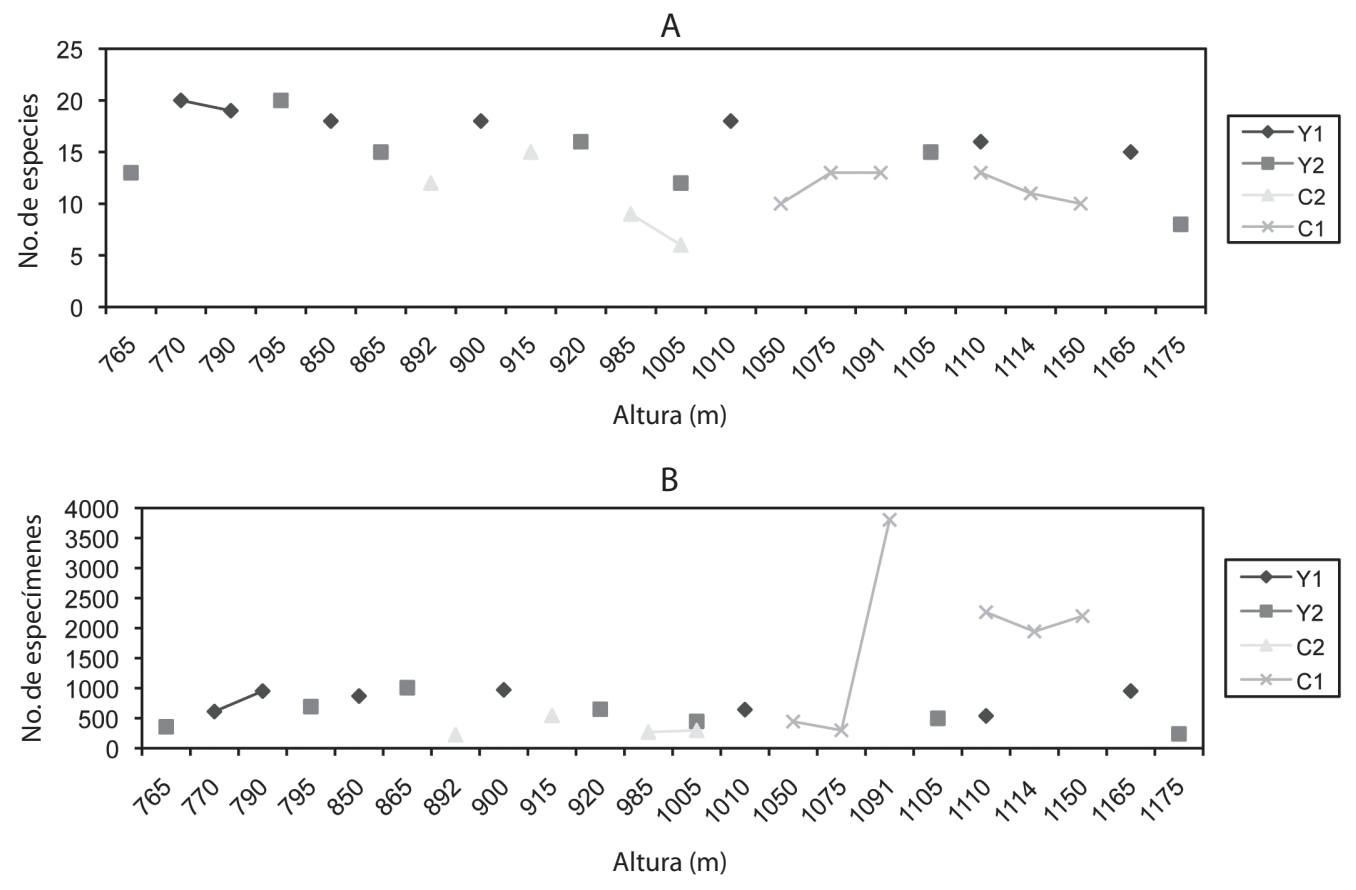

Fig. 2. Especímenes recolectados.

Fig. 2. Number of specimens collected.

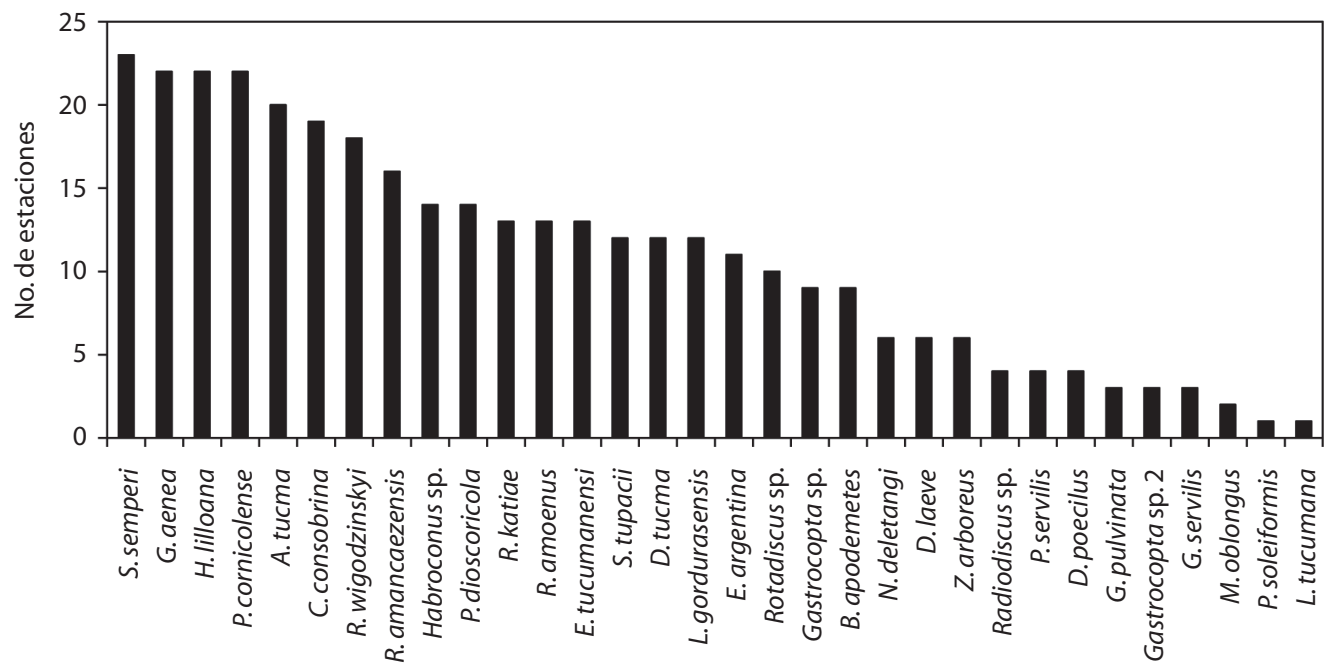

Especies

Fig. 3. Frecuencia de ocurrencia (parcelas en las que las especies fueron recolectadas).

Fig. 3. Frequency of occurrence (stations where the species were collected). 


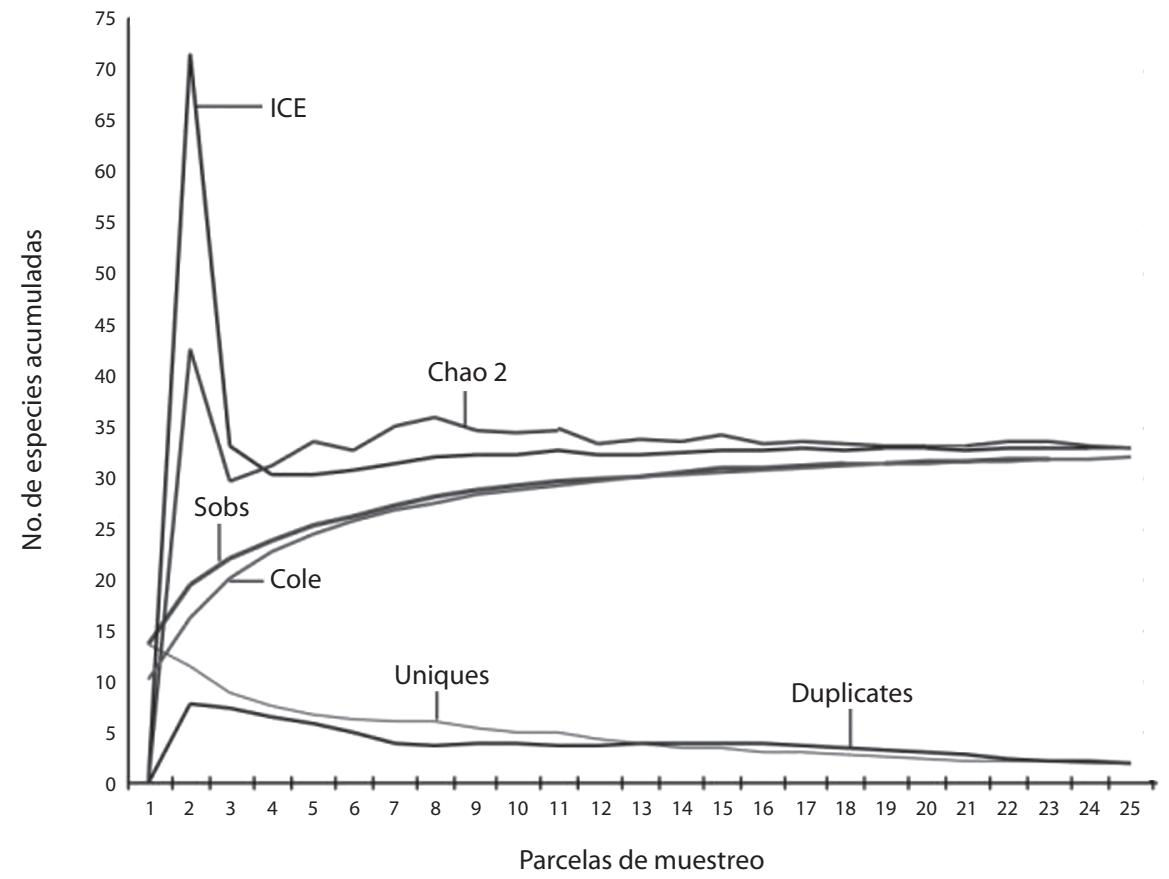

Fig. 4. Estimadores de riqueza basados en incidencia. Curvas de acumulación de especies y curva de Coleman obtenidas mediante EstimateS 5.0.1.

Fig. 4. Richness estimators based on incidence. Species accumulation curves and Coleman curves obtained with EstimateS 5.0.1.

seguida por la curva Chao2 es fluctuante al comienzo, sin embargo se estabiliza y unifica con la curva de ICE. La separación de las curvas Chao2 y la de Sobs fue disminuyendo a medida que aumentó el esfuerzo de muestreo (número de parcelas muestreadas) sin llegar a unirse. La curva de Coleman permaneció por debajo de la de Sobs, a la cual se unió posteriormente. Las curvas referidas a especies únicas "uniques" y de doble aparición "duplicates" (especies raras), mostraron un comportamiento similar (Fig. 4). La curva uniques presentó un descenso continuo y gradual, sin alcanzar el valor de cero. La curva duplicates inició con un ascenso y luego descendió aunque sin alcanzar el valor de cero.

El comportamiento de los estimadores no paramétricos utilizando datos de abundancia es mostrado en la Fig. 5. La curva de Sobs presenta un rápido crecimiento y se estabiliza formando una meseta. La curva del estimador ACE, muestra un ascenso inicial y luego comienza a aplanarse. Al comenzar la trayectoria de la curva ACE, la separación entre ella y la de Sobs es reducida, presentando luego un aumento para volver posteriormente a aproximarse, sin llegar a converger. La curva de Chao1 muestra un comportamiento similar a la de ACE con respecto a la curva de Sobs. La curva de Cole en un principio se mantiene distante de las curvas ACE, Chaol y Sobs y comienza a aproximarse a ellas a medida que aumenta el esfuerzo de muestreo. Puede apreciarse que las curvas de Sobs, ACE, Chaol y Cole, convergen en un punto. La curva de los doubletons muestra un marcado descenso que alcanza el valor de cero. 


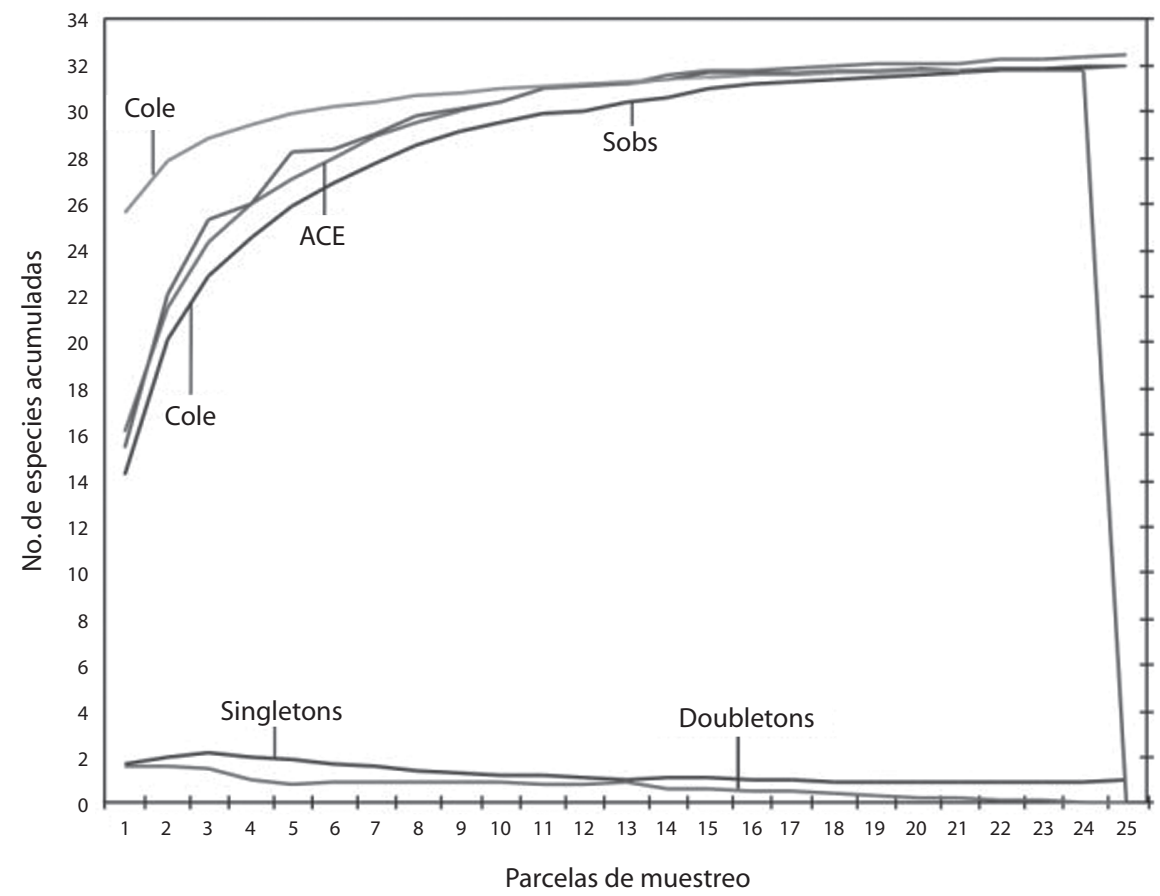

Fig. 5. Estimadores de riqueza basados en abundancia. Curvas de Acumulación de especies y curva de Coleman obtenidas mediante EstimateS 5.0.1.

Fig. 5. Richness estimators based on incidence. Species accumulation curves and Coleman curves obtained with EstimateS 5.0.1.

La curva de los singletons muestra un descenso gradual y continuo, sin llegar a cero. Estas curvas mantuvieron su separación al aumentar el número de muestras consideradas.

La diversidad $\alpha$ (Cuadro 5) calculada mediante el índice de Shannon fluctuó entre 1.5 (parcela 1) y 2.0 (parcela 23). El Índice de equitatividad (=Eveness) varió entre 0.3 (parcela 1) y 0.7 (parcela 25). El índice de Simpson varió entre 3.4 (parcela 1) y 4.5 (parcela 23 ) (Cuadro 5). Los valores de diversidad $\beta$, calculados a través del índice de Whittaker, fluctuaron entre 0.6 (parcela 1) y 4.3 (parcela 25) (Cuadro 5), con un valor promedio de 2.5. El índice de Jaccard indica que la mayor similitud en la composición taxonómica se presenta entre Y1 e Y2 (más del 65\%: 11 familias, 14 géneros y 21 especies compartidas), mientras que la menor similitud se presenta entre $\mathrm{C} 2$ y La Nina (menos del 25\%: cinco familias, seis géneros y seis especies compartidas). A su vez, entre Y1, Y2 y C1, el valor de similitud es alto (55$65 \%$ : ocho familias, diez géneros y 17 especies compartidas). Por otra parte, C2 sólo es similar a $\mathrm{C} 1$ y no al resto de las transectas (45-55\%: ocho familias, 11 géneros y 13 especies compartidas). La transecta de La Nina presentó mayor similitud con Y1 (45-55\%: siete familias, diez géneros y 13 especies compartidas) e Y2 (55-65\%: siete familias, diez géneros y 14 especies compartidas) mientras que la similitud con $\mathrm{C} 1$ fue de $35-45 \%$.

\section{DISCUSIÓN}

La riqueza de gasterópodos terrestres registrada en Selva de Yungas y Bosque Chaqueño 
CUADRO 5

Índices de diversidad

TABLE 5

Diversity indices

\begin{tabular}{|c|c|c|c|c|}
\hline Índice/parcela & Shannon (H') & Simpson (D) & Whittaker (I) & Evenness (E) \\
\hline 1 & 1.531 & 3.427 & 0.60 & 0.354 \\
\hline 2 & 1.773 & 3.961 & 0.68 & 0.417 \\
\hline 3 & 1.848 & 4.235 & 0.72 & 0.443 \\
\hline 4 & 1.902 & 4.508 & 0.72 & 0.456 \\
\hline 5 & 1.911 & 4.437 & 0.72 & 0.458 \\
\hline 6 & 1.931 & 4.449 & 1.00 & 0.483 \\
\hline 7 & 1.953 & 4.537 & 1.13 & 0.500 \\
\hline 8 & 1.971 & 4.572 & 1.46 & 0.532 \\
\hline 9 & 1.972 & 4.540 & 0.60 & 0.456 \\
\hline 10 & 1.975 & 4.546 & 1.13 & 0.506 \\
\hline 11 & 1.974 & 4.493 & 1.00 & 0.494 \\
\hline 12 & 1.986 & 4.540 & 1.67 & 0.554 \\
\hline 13 & 1.994 & 4.549 & 1.13 & 0.510 \\
\hline 14 & 1.996 & 4.553 & 3.00 & 0.665 \\
\hline 15 & 2.007 & 4.479 & 2.20 & 0.604 \\
\hline 16 & 1.998 & 4.534 & 2.55 & 0.630 \\
\hline 17 & 1.999 & 4.534 & 1.46 & 0.540 \\
\hline 18 & 2.004 & 4.541 & 1.46 & 0.541 \\
\hline 19 & 2.006 & 4.537 & 2.20 & 0.604 \\
\hline 20 & 2.007 & 4.533 & 1.46 & 0.542 \\
\hline 21 & 2.014 & 4.557 & 2.91 & 0.582 \\
\hline 22 & 2.018 & 4.570 & 1.67 & 0.562 \\
\hline 23 & 2.022 & 4.583 & 1.13 & 0.518 \\
\hline 24 & 2.019 & 4.561 & 2.55 & 0.637 \\
\hline 25 & 2.017 & 4.550 & 4.33 & 0.780 \\
\hline
\end{tabular}

Serrano del Parque Sierra de San Javier es de 32 especies. Dicha riqueza, se debe principalmente a la presencia de micromoluscos $(72 \%$ del total de especies), ya que los macromoluscos representan una pequeña proporción de la malacofauna (28\%). La riqueza de Stylommatophora (30 especies) fue superior, mientras que Systellommatophora y Caenogastropoda, estuvieron representadas por solo una especie cada una.

Comparaciones con estudios previos son difíciles de realizar debido a las diferentes metodologías empleadas, tamaño de áreas consideradas y a que no en todos los casos fueron recolectados micromoluscos y macromoluscos. La riqueza más aproximada a la obtenida en este trabajo, corresponde al estudio realizado por Gargominy \& Ripken (1998) quienes registraron 34 especies en $1 \mathrm{~km}^{2}$ de selva en Guyana Francesa en Sudamérica. Sin embargo, el área muestreada por Gargominy \& Ripken (1998) supera a la muestreada en el Parque San Javier. En el presente estudio fueron recolectadas 23 especies de micromoluscos en un 
área de igual tamaño al área de trabajo en la provincia de Tucumán de Salas Oroño et al. (2007). Estos autores ya señalaban la elevada riqueza de moluscos en la zona de San Javier que se confirma en el presente estudio. Miquel et al. (2007), realizaron una investigación sobre diversidad en el Sur de Brasil (Selva Paranaense), registrando 34 especies de micromoluscos. En dicho estudio se identificaron cinco géneros de Punctoidea Morse 1864, de los cuales cuatro fueron asimismo recolectados en el Parque San Javier (Noroeste Argentino). Existiría de acuerdo a Miquel et al. (2007) una fauna similar a nivel genérico de punctoideos entre las selvas de Yungas y Paranaense.

En nuestro estudio, se evidenció que la mayor riqueza de especies se ve favorecida con ciertas características del suelo y ambiente tales como la abundancia de hojarasca y palos podridos, elevada humedad y alto porcentaje de cobertura vegetal. Las parcelas de mayor riqueza pertenecen a la selva húmeda de las Yungas (700-850m). Por otro lado, el valor de riqueza más bajo se registró en bosque chaqueño $(1005 \mathrm{~m})$, un ambiente abierto, de suelo seco, compacto y agrietado, con poca hojarasca y abundantes rocas y palos secos.

Al comparar la riqueza entre parcelas de ambas ecorregiones a similares altitudes, se pudo observar que en Yungas la riqueza es superior a la de Chaco en todos los casos considerados. Así, por debajo de los $1000 \mathrm{~m}$ de altitud, en Yungas la riqueza varía entre 13 y 20 especies. A su vez, en Chaco dicha variación es entre nueve y 12 especies. Al considerar las parcelas ubicadas a una altitud superior a los 1 $000 \mathrm{~m}$, la variación en la riqueza fue de ocho18 especies y seis-13 especies, para Yungas y Chaco, respectivamente. Cabe destacar que la pendiente registrada en las parcelas tomadas en transectas de Chaco no fue tan pronunciada como en las de Yungas. La composición taxonómica de la comunidad de moluscos en Yungas no cambia con la altitud. En el Chaco serrano, se produce un cambio en la composición taxonómica entre las parcelas ubicadas a bajas altitudes (menos de $1000 \mathrm{~m}$ ) y aquellas de mayor altitud (más de $1000 \mathrm{~m}$ ). Cabrera
\& Willink (1973) aseguran que en la selva de Yungas tanto la precipitación como la temperatura disminuye a medida que aumenta la altitud sobre el nivel del mar. Los cambios de la riqueza de especies pueden deberse a las variaciones ambientales que experimentan. Los resultados aquí obtenidos, concuerdan con los de Salas Oroño et al. (2007) y Tattersfield et al. (2001), en cuyos estudios se registró una disminución de la riqueza con la altitud. Estos resultados son consistentes con el patrón general ampliamente observado en la mayoría de los grupos de animales y plantas (Rahbek 1995).

Aunque la región chaqueña según el presente estudio, tiene una riqueza total mayor que las Yungas, ambas Ecorregiones comparten el $53 \%$ de las especies. Dos especies que pueden habitar bosques húmedos, D. poecilus y $M$. oblongus, no fueron recolectadas en áreas de Yungas, solamente en la Ecorregión chaqueña. Asimismo, Salas Oroño et al. (2007), registran la presencia de Drymaeus Albers 1850 en selva de Yungas en su estudio. Si estos taxa hubieran sido registrados en nuestro estudio, la riqueza de las Yungas ascendería a 26 especies, superando así la riqueza de la región chaqueña.

Las especies recolectadas exclusivamente en la selva de Yungas fueron: S. tupacii, D. tucma, L. tucumana, Paralaoma servilis (Shuttleworth 1852), Zonitoides arboreus (Say 1916), D. laeve y P. soleiformis. Mientras que las especies recolectadas exclusivamente en el Bosque Chaqueño Serrano fueron: D. poecilus, B. apodemetes, Naesiotus deletangi (Parodiz 1946), Megalobulimus oblongus (Müller 1774), Gastrocopta pulvinata Hylton Scott 1948, G. servilis y Gastrocopta sp. 1 y sp. 2.

El muestreo cualitativo fue muy efectivo para la recolección de especímenes vivos aunque los mismos solo representan una pequeña proporción comparado con las conchas secas recolectadas. Esto motivó a que no se discriminara entre especímenes vivos y conchas secas en los análisis realizados. El muestreo cuantitativo resultó ser más eficaz para la recolección de micromoluscos, ya que a través de este método se registró mayor número de especies y de especímenes. Sin embargo, Emberton et 
al. (1996), en Madagascar, demostraron que el muestreo cualitativo fue mucho más eficiente que el cuantitativo, proporcionando un número de especímenes cerca de diez veces mayor y un número de especies siete veces mayor. Este resultado puede deberse a que en Emberton et al. (1996) el número de personas involucradas en la recolección cualitativa fue superior al de nuestro estudio. La elevada cantidad de especímenes que fueron recolectados en $\mathrm{C} 1$ por encima de los $1000 \mathrm{~m}$ de altitud, resulta llamativo por ser mayor que en Yungas a igual altitud. Por otro lado, en zona chaqueña por debajo de los $1000 \mathrm{~m}(\mathrm{C} 2)$ fue donde se recolectaron menos especímenes, probablemente por ser el área más seca, menos boscosa y con ausencia de hojarasca. La densidad fue elevada y varió entre 2 y 38 especímenes $/ \mathrm{m}^{2}$. Los valores de densidad fueron variables al considerar parcelas ubicadas a altitudes similares. Las parcelas correspondientes a Yungas, presentaron densidades superiores a los de la transecta La Nina (Salas Oroño et al. 2007) por debajo de los 1 $000 \mathrm{~m}$ de altitud, pero resultó ser ligeramente más elevada en La Nina en las parcelas por encima de este nivel altitudinal.

A. tucma y $S$. semperi son las especies de las que se recolectó mayor cantidad de especímenes. Ambas resultaron ser muy abundantes en Yungas en toda su extensión altitudinal y en zona chaqueña a altitudes superiores a $1000 \mathrm{~m}$, ya que por debajo de dicha altitud estuvieron pobremente representadas ó ausentes. Dichas especies igualmente resultaron ser las más abundantes en el estudio llevado a cabo por Salas Oroño et al. (2007). B. apodemetes, es la especie de macromoluscos más abundante, sobre todo en la zona chaqueña por debajo de 1 $000 \mathrm{~m}$. A su vez, S. tupacii fue la segunda especie de macromoluscos más abundante.

La familia con mayor representación fue Charopidae con siete especies, sobre un total de 26 especies citadas para Argentina (Fernández 1973). Estos datos coinciden con lo observado por Salas Oroño et al. (2007) en selvas de Tucumán, Barker \& Mayhill (1999) en Nueva Zelanda y por Miquel et al. (2007) en Brasil. Con respecto a Pupillidae, se registraron seis especies sobre el total de 15 citadas para Argentina (Fernández 1973). Orthalicidae, la familia de macromoluscos con mayor representación, se encuentra en el Parque Biológico de San Javier con cuatro especies sobre el total de 94 para Argentina (Fernández 1973).

Se registraron pocas especies raras, entre ellas, $P$. soleiformis, la cual estuvo representada por un único individuo. Solo tres especies, $M$. oblongus, L. tucumana y P. servilis estuvieron representadas por menos de diez individuos. Salas Oroño et al. (2007) registraron también como especie rara a L. tucumana. Por otra parte, P. servilis (=Radioconus pilsbryi Hylton Scott 1957) fue más abundante (46 especímenes) en Salas Oroño et al. (2007). Miquel et al. (2007), solamente recolectaron dos especímenes de $P$. servilis en su estudio llevado a cabo en la Selva Paranaense de Brasil.

Del total de especies recolectadas, se registraron algunas especies introducidas, tales como, Z. arboreus, P. servilis, C. consobrina y $D$. laeve. Es decir que en este estudio se registró un alto porcentaje de especies nativas (87\%). $Z$. arboreus fue recolectada exclusivamente en altitudes inferiores a $900 \mathrm{~m}$ en Yungas, por lo cual dicha especie podría estar relacionada con ambientes cercanos a zonas urbanas y por lo tanto, estaría en su primer estado de invasión (With 2001). Las restantes especies introducidas que se hallan en Yungas se extienden a mayores niveles altitudinales, estarían en su segundo estado de invasión, el de colonización (With 2001).

Al igual que en Salas Oroño et al. (2007), en el Parque San Javier las especies más frecuentes fueron: $S$. semperi, G. aenea y $H$. lilloana, mientras que las de menor frecuencia: P. soleiformis y L. tucumana.

La riqueza (métodos no paramétricos) fue aproximada o coincidente con la observada. Las curvas de Sobs, ICE, Chao2 y Cole, tienden a formar una asíntota a medida que aumenta el esfuerzo de muestreo, lo cual indica que aunque se aumente el esfuerzo de muestreo, no se incrementará el número de especies y por lo tanto, el muestreo realizado fue completo. Con respecto al comportamiento de las 
curvas uniques y duplicates, ambas tienden a descender hacia cero, comportamiento típico de un inventario completo. La curva de Coleman que detecta la agregación espacial en los datos, presentó los valores más similares a los observados, indicando que hay una distribución aleatoria de las especies raras.

Con respecto al comportamiento de los estimadores ACE, Chaol y Cole, sus curvas son asintóticas y presentan valores muy próximos a los observados, lo que indica que el muestreo realizado fue bueno. La curva de Coleman presenta una separación evidente con la curva de $S o b s$, lo que indica que las especies raras están agrupadas en pocas muestras (Colwell \& Coddington 1994). Los índices de Shannon y recíproco de Simpson, coincidieron en que la estación menos diversa es la número uno, mientras que la de mayor diversidad es la estación 23, existiendo menor equidad y mayor presencia de especies dominantes (Magurran 1989). En la parcela 23, la mayor parte de los especímenes recolectados son de las especies S. semperi ( $24 \%$ del total de los especímenes), S. tupacii $(24 \%)$ y C. consobrina (22\%). De este modo, cuanto mayor es el grado de dominancia de algunas especies y de rareza de las demás, menor es la biodiversidad (Halffter \& Ezcurra 1992). La estación 23 resulta con la mayor equidad y por lo tanto es la más diversa. En comparación con los resultados de Salas Oroño et al. (2007) las parcelas obtenidas en la transecta de La Nina, presentaron valores de Shannon superiores a los aquí obtenidos.

Según el índice de Shannon, la Ecorregión chaqueña es más diversa que la de Yungas. Llama la atención que las parcelas con mayor riqueza de especies (uno y nueve) presentan un índice de Equitatividad similar por lo que la composición de sus especies sería igualmente abundante. Por el contrario, la parcela con menor riqueza (25) presentó el mayor valor de Equitatividad.

En general existe una gran diferenciación en la composición de especies de las parcelas muestreadas ya que los valores obtenidos con el Índice de Whittaker son elevados, lo que puede ser el resultado de un reemplazamiento geográfico o ecológico de las especies. Las parcelas uno y dos (770-790m altitud), presentaron valores menores de Whittaker respecto a la 25, por lo que las primeras son similares en cuanto a composición de especies mientras que la última presenta la composición más distinta con respecto a las demás parcelas. Las parcelas de la Ecorregión Chaqueña presentan valores más elevados del índice de Whittaker respecto a la Ecorregión de Yungas lo que indica un incremento en la diferenciación en la composición de especies que presentan. A su vez, en la Ecorregión de Yungas es notable el aumento en la diversidad $\beta$ mostrado por las parcelas ubicadas a gran altitud. Las parcelas ubicadas entre 700 y $1000 \mathrm{~m}$ presentan mayor similitud en cuanto a composición de especies. De acuerdo al criterio de Winter \& Gittenberger (1998), las parcelas seis y 11 , con $\mathrm{I}=1$, tienen la misma fauna. Esto puede deberse a que ambas parcelas comparten características florísticas y se encuentran ubicadas en altitudes similares (900-1 100m).

Según el Índice de Jaccard, las transectas ubicadas en la Ecorregión de Yungas (Y1, Y2 y La Nina), son muy similares en cuanto a su composición. Dicha similitud podría estar relacionada con la semejanza en cuanto al ambiente, tipo de sustrato, vegetación, etc. A su vez, considerando las transectas pertenecientes a la Ecorregión Chaqueña ( $\mathrm{C} 1$ y $\mathrm{C} 2$ ), la similitud entre ambas es inferior a la presentada entre las transectas de Yungas. Cabe destacar que a pesar de pertenecer a la misma Ecorregión, ambas transectas son diferentes. Además, C1 tiene valores elevados de similitud con las transectas de Yungas. Por tal motivo, presenta una composición intermedia entre Yungas y Chaco Serrano.

A partir de los resultados obtenidos se destaca la importancia de realizar nuevos estudios, que permitan establecer específicamente las causas que determinan la distribución de los gasterópodos terrestres neotropicales. $\mathrm{La}$ falta de datos y disponibilidad de estudios de diversidad en distintas áreas de Sudamérica hace dudosa en la actualidad la hipótesis que la riqueza disminuye con el incremento de latitud en grupos de invertebrados terrestres no 
artrópodos, tales como moluscos, como se ha postulado para grupos de vertebrados. Teniendo en cuenta los datos disponibles sobre riqueza en gasterópodos terrestres éstos sugerirían la existencia de una riqueza similar en Guyana Francesa (Gargominy \& Ripken 1998) y sur de Brasil (Miquel et al. 2007) con la registrada en el Noroeste Argentino. Por lo tanto, sería de gran importancia la realización de estudios similares en otras zonas de bosques subtropicales y tropicales en Sudamérica que permitan la obtención de datos comparativos. Asimismo, el presente estudio confirma el hallazgo de una fauna de micromoluscos punctoideos similares a nivel genérico entre selvas Paranaense y de Yungas, ambos bosques húmedos subtropicales. Sin embargo, no existen datos concretos que permitan la comparación de grupos de macromoluscos para testar si esta similitud se mantiene, por lo que nuevos estudios sería de gran importancia.

El conocimiento actual de la malacofauna terrestre en Sudamérica tanto de la taxonomía, ecología y biogeografía es aún precario y fragmentado. La conservación de las especies y por ende de la biodiversidad se ve amenazada en la actualidad ante esta falta de conocimiento tan llamativa en grupos de invertebrados.

\section{AGRADECIMIENTOS}

Al Parque Biológico Sierra de San Javier, Universidad Nacional de Tucumán, por el permiso de investigación otorgado para llevar a cabo la colecta del material biológico. A Fátima Romero por la lectura crítica y los valiosos comentarios y sugerencias realizadas. Este trabajo fue financiado mediante el proyecto PIP 6048 de CONICET (Consejo Nacional de Investigaciones Científicas y Técnicas de Argentina) otorgado a M.G.C. M.J.M. es becaria y M.G.C. investigador de CONICET, Institución a la que se agradece su apoyo.

\section{RESUMEN}

Se realizó un inventario y se analizaron parámetros de diversidad de la malacofauna terrestre del Parque Biológico
Sierra de San Javier. Se llevaron a cabo muestreos cualitativos y cuantitativos en parcelas de $10 \times 10 \mathrm{~m}$ tomadas en transectas altitudinales, para un total de 22169 especímenes recolectados. Las identificaciones taxonómicas se llevaron a cabo a nivel de especie. Se construyó una matriz de especies por parcela para analizar patrones de diversidad y se utilizaron estimadores no paramétricos (ICE, ACE, Chao 1 y Chao 2) para calcular la diversidad del Parque, el grado de completitud del muestreo y la agregación espacial de los datos. Se calcularon los índices de Shannon, Simpson, Whittaker y Jaccard. La riqueza del Parque fue estimada en 32 especies distribuidas en 21 géneros y 13 familias. Solo una especie pertenece a Caenogastropoda, el resto son Pulmonados Stylommatophora y Systellommatophora. La familia más representada fue Charopidae mientras que la especie con mayor abundancia relativa fue Adelopoma tucma. La riqueza y diversidad fue levemente mayor en chaco seco que en bosque húmedo de Yungas. Los valores de diversidad obtenidos fueron elevados en comparación con estudios previos realizados en el noroeste Argentino.

Palabras clave: Stylommathophora, Systellommatophora. Yungas, Chaco Serrano, micromoluscos, macromoluscos, abundancia.

\section{REFERENCIAS}

Aktipis, S.W., G. Giribet, D.R. Lindberg \& W.F. Ponder. 2008. Gastropoda: An overview and analysis, p. 201237. In W.F. Ponder \& D.R. Lindberg (eds.). Phylogeny and Evolution of the Mollusca. Universidad de California, Berkeley, EEUU.

Barker, G.M. \& P.C. Mayhill. 1999. Patterns of diversity and habitat relationships in terrestrial mollusk communities of the Pukeamaru Ecological District, northeastern New Zeland. J. Biogeogr. 26: 215-238.

Barrientos, Z. 2003. Aspectos básicos sobre la clasificación, recolección, toma de datos y conservación de los moluscos. Rev. Biol. Trop. 51: 13-20.

Bouchet, P. \& J.P. Rocroi. 2005. Classification and nomenclator of Gastopod families. Malacologia 47: 1-397.

Brown, D.A., A. Grau, T. Lomáscolo \& N. Gasparri. 2002. Una estrategia de conservación para las selvas subtropicales de montaña (Yungas) de Argentina. Ecotrópicos 15: 147-159.

Cabrera, A.L. 1976. Regiones Fitogeográficas argentinas. Enciclopedia Argentina de Agricultura y Jardinería. Acme S.A.C.I., Buenos Aires, Argentina.

Cabrera, A.L. \& A. Willink. 1973. Biogeografía de América Latina. Programa Regional de Desarrollo Científico y Tecnológico. Departamento de Asuntos Científicos. 
Secretaria Gral. de la Organización de los Estados Americanos, Serie Biológica 13, Washington D.C., EEUU.

Cameron, R.A.D., M. Mylonas, K. Triantis, A. Parmakelis \& K. Vardinoyannis. 2003. Land snail diversity in square kilometer of Cretan maquis: modest species richness, high density and local homogeneity. J. Moll. Stud. 69: 93-99.

Cameron, R.A.D., R.M.T. Da Cunha \& A.M. Frias Martins. 2007. Chance and necessity: Land snail faunas of Sao Miguel, Azores, compared with those of Madeira. J. Moll. Stud. 73: 11-21.

Chao, A. 1984. Non-parametric estimation of the number of classes in a population. Scand. J. Statist. 11: 265-270.

Chao, A. 1987. Estimating the population size for capturerecapture data with nequal catchability. Biometrics 43: 783-791.

Chao, A., M.C. Ma \& M.C.K. Yang. 1993. Stopping rules and estimation for recapture debugging with unequal failure rates. Biometrika 80: 193-201.

Coleman, B.D. 1981. On random placement and speciesarea relations. Math. Biosci. 54: 191-215.

Coleman, B.D., M.A. Mares, M.R. Willig \& Y.H. Hsieh. 1982. Randomness, area, and species richness. Ecology 63: 1121-1133.

Colwell, R.K. 1997. EstimateS: Statistical estimation of species richness and shared species from samples. Version 5.0.1. User's guide and application (También disponible en línea: http://viceroy.eeb.uconn. edu/estimates).

Colwell, R.K. \& J.A. Coddington. 1994. Estimating terrestrial biodiversity through Extrapolation, p. 101-118. In D.L. Hawsworth (ed.). Biodiversity Measurement and its Estimation. Chapman \& Hall, Londres, Inglaterra.

de Winter, A.J. \& E. Gittenberger. 1998. The land snail fauna of a square kilometer patch of rainforest in Southwestern Cameroon: High species richness, low abundance and seasonal fluctuations. Malacologia 40: $231-250$.

Emberton, K.C., T.A. Pearce \& R. Randalana. 1996. Quantitatively sampling land-snail species richness in Madagascan rainforests. Malacologia 38: 203-212.

Emberton, K.C., A. Pearce \& R. Randalana. 1999. Molluscan diversity in the unconserved Vohimena and the conserved Anosy mountain chains, Southeast Madagascar. Biol. Conserv. 89: 183-188.
Fernández, D. 1973. Catálogo de la malacofauna terrestre argentina. Comisión de Investigaciones Científicas de la Provincia de Buenos Aires. 4: 1-197.

Fernández, D. \& Z.A. Castellanos. 1973. Clave genérica de la malacofauna terrestre argentina. Rev. Mus. La Plata (N. S.), Zool. 11: 265-285.

Fontaine, B., O. Gargominy \& E. Neubert. 2007. Land snail diversity of the Savanna/Forest mosaic in Lopé National Park, Gabon. Malacologia 49: 313-338.

Gargominy, O. \& T.E.J. Ripken. 1998. Micro-pulmonates in tropical rainforest litter: A new bio-jewel, p. 116. In R. Bieler \& P.M. Mikkelsen (eds.). Abstract of the World Congress of Malacology, Washington D.C., EEUU. Field Museum of Natural History, Chicago, EEUU.

Halffter, G. \& E. Ezcurra. 1992. ¿Qué es la biodiversidad?, p. 3-24. In G. Halffter (ed.). La Diversidad biológica de Iberoamérica. Acta Zool. Instituto de Ecología, SEDESOL \& CYTED-D, Xalapa, Veracruz, México.

Hausdorf, B. 2006. The systematic position of Scolodonta Doring, 1875 and Scolodontidae H. B. Baker, 1925 (Gastropoda:Pulmonata). Zool. Anz. 245: 161-165.

Hausdorf, B. 2007. Revision of the American Pupisoma species (Gastropoda: Pupilloidea). J. Nat. Hist. 41: 1481-1511.

Heyer, W.R., J. Coddington, W.J. Kress, P. Acevedo, D. Cole, T.L. Edwin, B.J. Meggers, M.G. Pogue, R.W. Thorington, R.P. Varoi, M.J. Weitzman \& S.H. Weitzman. 1999. Amazonian biotic data and conservation decisions. Cienc. Cult. (San Pablo) 51: 372-385.

Hylton Scott, M.I. 1948. Moluscos del Noroeste Argentino. Acta Zool. Lilloana 6: 241-274.

Hylton Scott, M.I. 1950. Sobre la presencia del Género Pupisoma en la Argentina (Pulmonata: Pupillidae). Neotropica 6: 25-29.

Hylton Scott, M.I. 1957. Endodontidos Neotropicales II. (Moll, Pulm.). Neotropica 3: 79-87.

Hylton Scott, M.I. 1984. Anotaciones sobre el Género Guppya Morch, 1867 (Moll. Pulmonata). Neotropica 30: $89-96$.

Krebs, C.J. 1989. Ecological methodology. Harper \& Row, Nueva York, EEUU.

Krebs, C.J. 1995. Estudio de la Distribución y la Abundancia. Instituto Ecológico de Recursos Animales, Universidad de Columbia Británica, Canadá. 
Lee, S.M. \& A. Chao. 1994. Estimating population size via sample coverage for closed capture-recapture models. Biometrics 50: 88-97.

Lydeard, C., R. Cowie, W.F. Ponder, A.E. Bogan, P. Bouchet, S.A. Clark, K.S. Cummings, T.J. Frest, O. Gargominy, D.G. Herert, R. Hershler, K.E. Perez, B. Roth, M. Seddon, E.E. Strong \& F.G. Thompson. 2004. The global decline of nonmarine mollusks. BioScience 54: 321-330.

Magurran, A.E. 1989. Diversidad Ecológica y su medición Vedra, Barcelona, España.

Margalef, R. 1972. Homage to Evelyn Hutchinson, or why is there an upper limit to Diversity, p. 213-235. In E.S. Deevey (ed.). Growth by Intussusception. Ecological Essays in honor of G.E. Hutchinson. Trans. Connect. Acad. Arts. Sci. 44: 211-235.

Miquel, S.E., R. Ramirez \& J.W. Thomé. 2004. Lista preliminar de los Punctoideos de Río Grande do Sul, Brasil, con descripción de dos especies nuevas (Mollusca, Gastropoda, Stylommatophora). Rev. Brasil. Zool. 21: 925-935.

Miquel, S.E. \& E.S. Bellosi. 2006. MicroGasterópodos terrestres (Charopidae) del Eoceno Medio de Gran Barranca (Patagonia Central, Argentina). Ameghiniana 44

Miquel S.E., R. Ramirez \& J.W. Thomé. 2007. Biodiversidad y taxonomía de micromoluscos Punctoidea del sur de Brasil, con la descripción de una nueva especie de Radiodiscus de la Mata Atlántica (Mollusca, Gastropoda, Pulmonata). Rev. Mus. Argent. Cienc. Nat. 9: 205-230.

Morrone, J.J. 2004. Panbiogeografía, componentes bióticos y zonas de transición. Rev. Brasil. Entomol. 48: $149-162$

Rahbek, C. 1995. The elevational gradient of species richness: a uniform pattern? Ecography 18: 200-2005.
Salas Oroño, E., M.G. Cuezzo \& F. Romero. 2007. Land snail diversity in subtropical rainforest mountains (Yungas) of Tucumán, Northwestern Argentina. Am. Malacol. Bull. 22: 17-26.

Schilthuizen, M. \& H.A. Rutjes. 2001. Land snail diversity in a square kilometer of tropical rainforest in Sabah, Malaysian Borneo. J. Moll. Stud. 67: 417-423.

Solem, A. 1984. A world model of land snail diversity and abundance, p. 6-22. In A. Solem \& A.C. van Bruggen (eds.). Worls-wide snails: Biogeographical studies on non-marine Mollusca. E.J. Brill/W. Backhuys, Leiden, Holanda.

Tattersfield, P.C., C.M. Warui, M.B. Seddon \& J.W. Kiringe. 2001. Land-snail faunas of afromontane forest of Mount Kenya, Kenya: Ecology, diversity and distribution patterns. J. Biogeogr. 28: 843-861.

Toti, D.S., F.A. Coyle \& J.A. Miler. 2000. A structured inventory of appalachian grass bald and heath bald spider assemblages and a test of species richness estimator performance. J. Arachnol. 28: 329-345.

Valdora, E.E. \& M.B. Soria. 1999. Árboles de interés forestal y ornamental para el Noroeste argentino. Laboratorio de Investigaciones Ecológicas de las Yungas (LIEY), Universidad Nacional de Tucumán, Tucumán, Argentina.

Vides Almonacid, R., H.R. Ayarde, G.J. Scrocchi, F. Romero, C. Boero \& J.M. Chani. 1998. Biodiversidad de Tucumán y el Noroeste argentino. Opera Lilloana 43: 1-89.

Weyrauch, W.W. 1965. Cinco nuevos Endodontidos de Argentina y Perú (Gastropoda, Euthyneura). Neotropica 11: 105-115.

Whittaker, H.R. 1975. Evolution of species diversities in land communities. Evol. Biol. 10: 1-68.

With, K.A. 2001. The landscape ecology of invasive spread. Conserv. Biol. 16: 1192-1203. 